\title{
Video Article \\ Long-term Intravital Immunofluorescence Imaging of Tissue Matrix Components with Epifluorescence and Two-photon Microscopy
}

\author{
Esra Güç* ${ }^{1}$, Manuel Fankhauser* ${ }^{1}$, Amanda W. Lund ${ }^{1,2}$, Melody A. Swartz ${ }^{1}$, Witold W. Kilarski ${ }^{1}$ \\ ${ }^{1}$ Institute of Bioengineering and Swiss Institute of Experimental Cancer Research (ISREC), École Polytechnique Fédérale de Lausanne \\ ${ }^{2}$ Department of Cell and Developmental Biology and Knight Cancer Institute, Oregon Health \& Science University \\ * These authors contributed equally
}

Correspondence to: Witold W. Kilarski at witold.kilarski@epfl.ch

URL: https://www.jove.com/video/51388

DOI: doi: $10.3791 / 51388$

Keywords: Bioengineering, Issue 86, Intravital imaging, epifluorescence, two-photon imaging, Tumor matrix, Matrix remodeling

Date Published: 4/22/2014

Citation: Güç, E., Fankhauser, M., Lund, A.W., Swartz, M.A., Kilarski, W.W. Long-term Intravital Immunofluorescence Imaging of Tissue Matrix Components with Epifluorescence and Two-photon Microscopy. J. Vis. Exp. (86), e51388, doi:10.3791/51388 (2014).

\section{Abstract}

Besides being a physical scaffold to maintain tissue morphology, the extracellular matrix (ECM) is actively involved in regulating cell and tissue function during development and organ homeostasis. It does so by acting via biochemical, biomechanical, and biophysical signaling pathways, such as through the release of bioactive ECM protein fragments, regulating tissue tension, and providing pathways for cell migration The extracellular matrix of the tumor microenvironment undergoes substantial remodeling, characterized by the degradation, deposition and organization of fibrillar and non-fibrillar matrix proteins. Stromal stiffening of the tumor microenvironment can promote tumor growth and invasion and cause remodeling of blood and lymphatic vessels. Live imaging of matrix proteins, however, to this point is limited to fibrillar collagens that can be detected by second harmonic generation using multi-photon microscopy, leaving the majority of matrix components largely invisible. Here we describe procedures for tumor inoculation in the thin dorsal ear skin, immunolabeling of extracellular matrix proteins and intravital imaging of the exposed tissue in live mice using epifluorescence and two-photon microscopy. Our intravital imaging method allows for the direct detection of both fibrillar and non-fibrillar matrix proteins in the context of a growing dermal tumor. We show examples of vessel remodeling caused by local matrix contraction. We also found that fibrillar matrix of the tumor detected with the second harmonic generation is spatially distinct from newly deposited matrix components such as tenascin C. We also showed long-term (12 hours) imaging of T-cell interaction with tumor cells and tumor cells migration along the collagen IV of basement membrane. Taken together, this method uniquely allows for the simultaneous detection of tumor cells, their physical microenvironment and the endogenous tissue immune response over time, which may provide important insights into the mechanisms underlying tumor progression and ultimate success or resistance to therapy.

\section{Video Link}

The video component of this article can be found at https://www.jove.com/video/51388/

Introduction

Intravital imaging of inflammatory, metastatic and matrix remodeling processes has been an important area of research and has motivated the creation of numerous transgenic reporter mouse models that express fluorescent proteins ${ }^{1,2}$. Due to out-of-focus fluorescent signals, which reduce image quality and limits light penetration through the tissue, imaging thick tissue is possible only with confocal or multi-photon scanning microscopy ${ }^{3}$. Using faster, less expensive and complex epifluorescence microscopy is possible only with nearly two-dimensional tissues such as the chicken chorio-allantoic membrane ${ }^{4}$ or mouse ear dermis ${ }^{5}$. Most imaging systems take advantage of transgenic mice expressing various fluorescent proteins in a cell type-specific fashion. Even though these proteins offer weak phototoxicity, they induce immune response ${ }^{6}$. Furthermore, it is difficult to switch between specific cell subtypes or mark their basal or activated states as such change requires the preparation of a new genetic model. In addition, as fluorescent protein expression is generally restricted to intracellular compartment, it is usually not possible to image extracellular structures like basement membrane proteins or tissue chemokine deposits ${ }^{7}$. Instead, indirect labeling with antibodies against extracellular antigens offers flexibility for virtually any cell-type or matrix specific component ${ }^{8,9}$. However, the major disadvantage of this labeling approach is associated with immunotoxicity mediated by antigen-antibody immune complexes that can trigger complement systemdependent cell toxicity and phagocytosis of cells and extracellular structures ${ }^{10}$.

The extracellular matrix of the tumor microenvironment, but also of normal tissue during inflammation or wound healing, undergoes substantial remodeling. Stromal stiffening of the tumor microenvironment can promote tumor growth and invasion due to stress-induced signaling mechanisms and cause remodeling of blood and lymphatic vessels ${ }^{11}$. However, live imaging of matrix proteins is limited to fibrillar collagens that can be detected by second harmonic generation using multi-photon microscopy. Recently we have published a novel intravital imaging method that minimizes the risk of photo- and immune-toxic damage to imaged cells and tissue structures ${ }^{5}$. In comparison with established imaging techniques where the single round of continuous intravital visualization is in a range of 30 minutes to 2 hours ${ }^{12-17}$, our intravital immunofluorescence (IF) technique allowed 12 hours (long-term ${ }^{8}$ ) imaging. It is important to note that the imaging time is restricted to 12 hours due to limits defined in our animal protocol but there are no technical counter-indications that it cannot be prolonged if critical health parameters 
of the animal, like blood pressure and heart rate are controlled ${ }^{8}$. In addition, by using an automated fluorescence stereomicroscope we were able to collect images from multiple fields during a single experiment and observed rare immunological and remodeling processes that occurred in the physiological context of the skin supported by functional blood and lymphatic vessels. Because the stereomicroscopic lens has a relatively large, $2 \mathrm{~cm}$, working distance and in contrast to two-photon microscopic optics does not require vaporizing water immersion, long-term imaging was performed only under fluorescence stereomicroscope. Intravital IF allowed the immune labeling and detection of any extracellular matrix component of the normal skin. The design of this technique is based on the innovative concept of using immunostaining for live cells and tissue matrix elements on surgically exposed mouse dermis without causing harmful immunotoxic effects ${ }^{5}$. The surgical procedure itself is safe to the dermis vasculature as it relies only on the separation of two skin layers in the ear that are independently innervated, autonomously fed by blood and drained by separate lymphatic circulations. Our experimental setup allows imaging of a range of important patho-physiological events over at least 12 hours, including leukocyte trafficking between blood and lymphatic vessels and wound healing processes. In the original publication, we compared our technique to state-of-the-art standards in various fields of intravital imaging. Consequently, we observed blood vessel extravasation and lymphatic intravasation events by various types of leukocytes, including the unique visualization of immune cells entering collecting instead of expected initial lymphatic vessels ${ }^{15}$. Also, complementing the observations done by other groups ${ }^{9,18}$, we found that in vivo CCL21 is able to form strong, discontinuous deposits on collecting but only infrequently on initial lymphatics.

Here we describe a modified intravital IF technique that can be combined with two-photon microscopy to detect network of fibrillar collagens using second harmonic generation (SHG). We show that this method can also be used for imaging cancer cell invasion in context of the dynamic tumor microenvironment, which includes matrix molecules such as tenascin $C$,that are largely unexplored by current imaging techniques ${ }^{19}$. We found that the fibrillar matrix of the tumor, detected with the second harmonic generation, occupies different locations of the tumor microenvironment than the newly deposited matrix composed of tenascin $\mathrm{C}$. Furthermore, we describe examples of vessel remodeling caused by local matrix contraction, long-term (12 hours) imaging of T-cell interactions with tumor cells and tumor cell migration along collagen IV of the basement membrane. Such events can be imaged while simultaneously recording local physiological parameters such as blood vessel permeability and lymphatic drainage.

\section{Protocol}

All procedures performed on animals were in strict accordance with the Swiss Animal Protection Act, the ordinance on animal protection and the ordinance on animal experimentation. We confirm that our Institutional Animal Care and Use Committee (IACUC), named Commission de Surveillance de l'Etat de Vaud (Permit Number: 2687), specifically approved this study.

\section{Tumor Inoculation of the Ear}

1. Culture B16-F10-GFP melanoma cells in a $10 \mathrm{~cm}$ Petri dish using DMEM $+10 \%$ FBS media.

2. When the cells are $80-90 \%$ confluent, wash them with PBS and detach them by trypsinization. Spin down cells at $1,500 \times \mathrm{g}$ for 5 min at $4{ }^{\circ} \mathrm{C}$, resuspend the pellet in $1 \mathrm{ml}$ Ringer's solution and transfer it into a $1.5 \mathrm{ml}$ Eppendorf tube. Spin again and remove all supernatant except a thin layer of medium that stays just above the pellet. Resuspend the cells to end up with a thick cell slurry.

3. Anesthetize the mouse with a mixture of ketamine/dorbene [medetomidine] $(75 \mathrm{mg} / \mathrm{kg}-1 \mathrm{mg} / \mathrm{kg}$ ) and confirm that the animal is sufficiently anesthetized by performing a gentle toe pinch. Increase the isoflurane concentration in steps of $0.1 \%$ in case movements are observed (e.g. withdrawal of the paw). Keep mouse on a rectal thermistor controlled heating pad $\left(37^{\circ} \mathrm{C}\right)$ during the whole procedure and protect its eyes with an appropriate ophthalmic ointment.

4. Prepare a Hamilton syringe ( $33 \mathrm{G}$ needle, $10 \mathrm{ml}$ syringe volume). Remove the tip cap with the needle and load $20 \mu \mathrm{l}$ of cell slurry on top of the tip chamber. Pull back the plunger until $5 \mathrm{ml}$ slurry is loaded inside the syringe. Remove the excess slurry from the tip chamber and close the tip cap.

5. Using adhesive tape, fix the proximal edge of the ear on the tip of an index finger. In a $45^{\circ}$ angle, slowly insert the Hamilton syringe needle between dorsal dermis and the cartilage. Once inside, penetrate the ear proximal to distal for about 2-3 mm.

Note: The procedure of cell injection should be done by trained and certified technician. In addition this process must be perform slowly and in sequence: first the needle should be placed horizontally to the ear skin and inserted slowly into the ear dermis. Once and only then the needle end is in the skin, the plunger should be pressed. Pressing of the plunger will initiate cells inoculation in the skin. In the unlikely event of the needle passing the two layers of the ear dermis, the layer of the cartilage and enters the skin of the person performing the experiment, the pain inflicted to the experimentator finger should be considered as an alarming sign and cause immediate retraction of the needle from the experimentator skin. In that case plunger should not be pressed and skin should be sterilized with alcohol. Alternatively, inanimate object instead of a finger can be used to stabilize the ear.

6. Inject $3 \mu \mathrm{l}$ cell slurry and slowly retract the needle from the ear. Let tumor cells form a solid tumor during 7-9 days and follow the tumor growth by using a fluorescence stereomicroscope.

7. Alternatively, to follow the early steps of tumor cell interaction with tissue matrix, apply $50 \mu \mathrm{l}$ of 100,000 tumor cells suspended in Ringer's solution on the exposed and stained ear dermis (after step 3.3).

\section{Ear Surgery}

1. Three days before the experiment, shave the mouse head, depilate the hairs around the head and ear (10-15 sec) and rinse with water.

2. Anesthetize the mouse with a mixture of humidified oxygen and isoflurane (3-4\% induction, $1-2 \%$ maintenance) and confirm that the animal is sufficiently anesthetized by performing a gentle toe pinch. Increase the isoflurane concentration in steps of $0.1 \%$ in case movements are observed (e.g. withdrawal of the paw). Keep mouse on a rectal thermistor controlled heating pad $\left(37^{\circ} \mathrm{C}\right)$ during the whole procedure and protect its eyes with an appropriate ophthalmic ointment.

3. Build a platform made out of 8 glued glass histology slides. Turn the mouse on its back and gently place the tumor-bearing ear on the stack of glass slides. Use small stripes of adhesive tape to fixate the anterior and posterior edges of the ear to the stack.

4. Cut the ventral skin of the ear along the antihelix of the mouse pinna using a scalpel. With the help of curved tweezers, gently peel off the ventral dermis and the cartilage from the dorsal dermis where the tumor was inoculated. Pull off the ventral skin and cartilage with curved 
forceps, exposing the dorsal dermis. NOTE: If the major vessels of the ear are cut or the blood circulation does not return to normal flow within $15 \mathrm{~min}$, the ear cannot be used for imaging. Always keep the opened ear skin wet by using Ringer's buffer and protect the humidity by using a coverslip.

5. In case there is persistent bleeding form tumor vessels, stop the bleeding by adding $100 \mu$ thrombin (5 U/ml) in Ringer's buffer (102 $\mathrm{mM}$ $\mathrm{NaCl}, 5 \mathrm{mM} \mathrm{KCl}, 2 \mathrm{mM} \mathrm{CaCl}, 28 \mathrm{mM}$ sodium lactate) on top of the ear for $5 \mathrm{~min}$.

6. Wash the ear twice with approximately $5 \mathrm{ml}$ of Ringer's buffer and remove extra liquid with sterile wipes. Immediately proceed to the next step (do not let the open ear dry at any point!).

\section{Immunofluorescence Staining}

Use Ringer's buffer supplemented with human serum (1:10), mouse polyclonal secondary antibody to human lgG (1:50), and $125 \mathrm{IU} / \mathrm{ml}(2.5 \mathrm{mg} /$ $\mathrm{ml}$ ) aprotinin (blocking buffer) for all staining steps. Aprotinin inhibits plasmin promoting coagulation to limit initial bleeding that may occur after surgery.

1. Fold the part of the ear with the open dermis into the eminentia conchae and dry the outer unopened ear dermis with sterile wipes. Immobilize the ear on the stack of glass slide by applying $0.5 \mu \mathrm{l}$ of surgical glue to the anterior and posterior dorsal edges. Then, gently flatten the dorsal ear dermis onto the glass.

2. Apply primary antibodies targeting extracellular matrix molecules at a concentration of $10 \mu \mathrm{g} / \mathrm{ml}$ in a total volume of $100 \mu \mathrm{l}$ blocking buffer to the exposed ear. Cover the ear with a cover slip in order to prevent the staining solution to dry on the ear edges. Incubate for 15 min. Wash the ear twice with approximately $5 \mathrm{ml}$ of Ringer's buffer.

3. Apply appropriate secondary antibodies or streptavidin conjugates (fluorophores with a high excitation wavelength such as $594 \mathrm{~nm}$ or $647 \mathrm{~nm}$ are favorable for intravital imaging) at a concentration of $10 \mu \mathrm{g} / \mathrm{ml}$ in a total volume of $100 \mu \mathrm{l}$ blocking buffer to the exposed ear. Cover the ear with a cover slip and incubate for $15 \mathrm{~min}$. Wash the ear twice with approximately $5 \mathrm{ml}$ of Ringer's buffer.

\section{Interaction of Blood-borne Activated Splenocytes with Tumor Cells in situ}

1. 7 days after inoculation of GFP-B16-F10 melanoma on the back skin of congenic mouse, euthanize the animal and collect spleens. Isolate splenocytes with mechanical disruption of the spleen through a $70 \mu \mathrm{m}$ cell strainer.

2. Label splenocytes for $8 \mathrm{~min}$ at $37^{\circ} \mathrm{C}$ with Red CMTPX cell cytoplasm stain ( $1 \mu \mathrm{M}$ in PBS), wash 4 times in $15 \mathrm{ml}$ at $4{ }^{\circ} \mathrm{C}$ and immediately inject with $200 \mu \mathrm{l}$ serum free medium into the tail vein of mouse whose ears were inoculated with B16-F10- GFP 7 days earlier.

\section{Intravital Imaging by Using a Stereomicroscope}

1. For short-term imaging (up to $2 \mathrm{hr}$ ), add freshly prepared sterile ascorbate-Ringer's buffer containing $140 \mathrm{mM}$ sodium ascorbate, $10 \mathrm{mM}$ HEPES, $4 \mathrm{mM} \mathrm{KCl}$ and $5 \mathrm{mM} \mathrm{CaCl}_{2}$, at a pH of 7.5 (ascorbate-Ringer's buffer final osmolarity $320 \mathrm{mOsM}$ ) on top of the immobilized ear. Cover the ear with a coverslip and start imaging by using a fluorescence stereomicroscope with $2 \mathrm{X}$ lens.

2. For long-term imaging (more than $2 \mathrm{hr}$ ), place the outlet of a needle (connected to a reservoir containing ascorbate-Ringer's buffer) under the coverslip, about $0.5 \mathrm{~cm}$ away from the ear. Use a peristaltic pump at a speed of $1 \mu \mathrm{l} / \mathrm{min}$ to constantly deliver ascorbate-Ringer's buffer to the chamber under the coverslip.

3. Change the $1 X$ lens (working distance $6 \mathrm{~cm}$ ) to $2 X$ lens (working distance $2 \mathrm{~cm}$ ). Open the acquisition software and configure the settings of the fluorescent stereomicroscope. In camera settings choose 12 bit as the image color depth and adjust the camera range of grey-scale values from $0 \%$ (minimum) to $5.1 \%$ (maximum) and set gamma correction to 2 .

1. Set acquisition gain to 1 (minimum), fluorescence intensity to 1,000 (maximum), magnification set to $280 \mathrm{X}-320 \mathrm{X}$ and adjust the exposure time avoiding over- or under- exposure (exposure time should be less than $1 \mathrm{sec}$ ). Depending on the number of imaging fields (usually 10 to 20), time interval for acquisition cycle should be set between 1 to $2 \mathrm{~min}$. Shorten times may cause the bleaching and phototoxicity.

4. Choose several fields that contain tumor cells as well as stained ECM proteins (such as in Figure 3). Using the motorized stage, acquire images of the appropriate fluorescent channels (such as GFP and fluorophore 647 in Figure 3) over time (e.g. every 2 min).

5. After the experiment, euthanize the mouse according to institutional animal guidelines. In this case, at the end of the experiment, euthanize the anesthetized mouse by cervical dislocation followed by exsanguination (intracardiac perfusion).

\section{Intravital Imaging by Using a Multiphoton Microscope}

1. Using silicon grease, build a circular wall of about $2 \mathrm{~cm}$ diameter and 2-3 mm height around the ear, starting at the base of the ear. Make sure there are no leaking points. Fill the circle with ascorbate-Ringer's buffer.

2. Place mouse on the stage and connect the heating pad $\left(37^{\circ} \mathrm{C}\right)$.

3. Open acquisition software and configure the settings of the multiphoton microscope.

4. Choose up to four different fields that contain tumor cells and stained ECM proteins (such as in Figure 3). In this example, tune the TiSaphire laser to $850 \mathrm{~nm}$ for GFP signal a subsequent single photon 647 for fluorophore 647 staining and visualize fibrillar collagens with SHG. Acquire images of the appropriate fluorescent channels (such as GFP and fluorophore 647 in Figure 3) and second harmonic generation over time, e.g. every $2 \mathrm{~min}$, with water immersion HCX APO 20X with 1.00 NA lens and 2 mm working distance.

5. After the experiment, euthanize anesthetized mouse with cervical dislocation followed by exsanguination (intracardiac perfusion). 


\section{Representative Results}

To date, immunostaining in live tissue is not typically used due to formation of immune complexes leading to high staining background and immunotoxicity ${ }^{20}$. This was overcome by pre-blocking Fcy receptors on tissue macrophages, thus "blinding" these cells to subsequent indirect strong immunostaining. As the labeling was extracellular, phototoxicity and fluorophore bleaching could be controlled by immersion of the tissue in natural antioxidant buffered, isosmotic ascorbic acid (Fig. 1A). Since our initial description of this method ${ }^{5}$, we improved our anti-bleaching and anti-phototoxic technique so that photobleaching can now not only be inhibited but completely prevented (Fig. 1B). This is done by embedding the ear in a large volume of antioxidant $(100 \mu \mathrm{l})$ within the chamber where the ear is immobilized. Note that 300 seconds constant imaging time corresponds to 10 hours of imaging when pictures are collected for 500 msec every one minute (the average imaging settings).
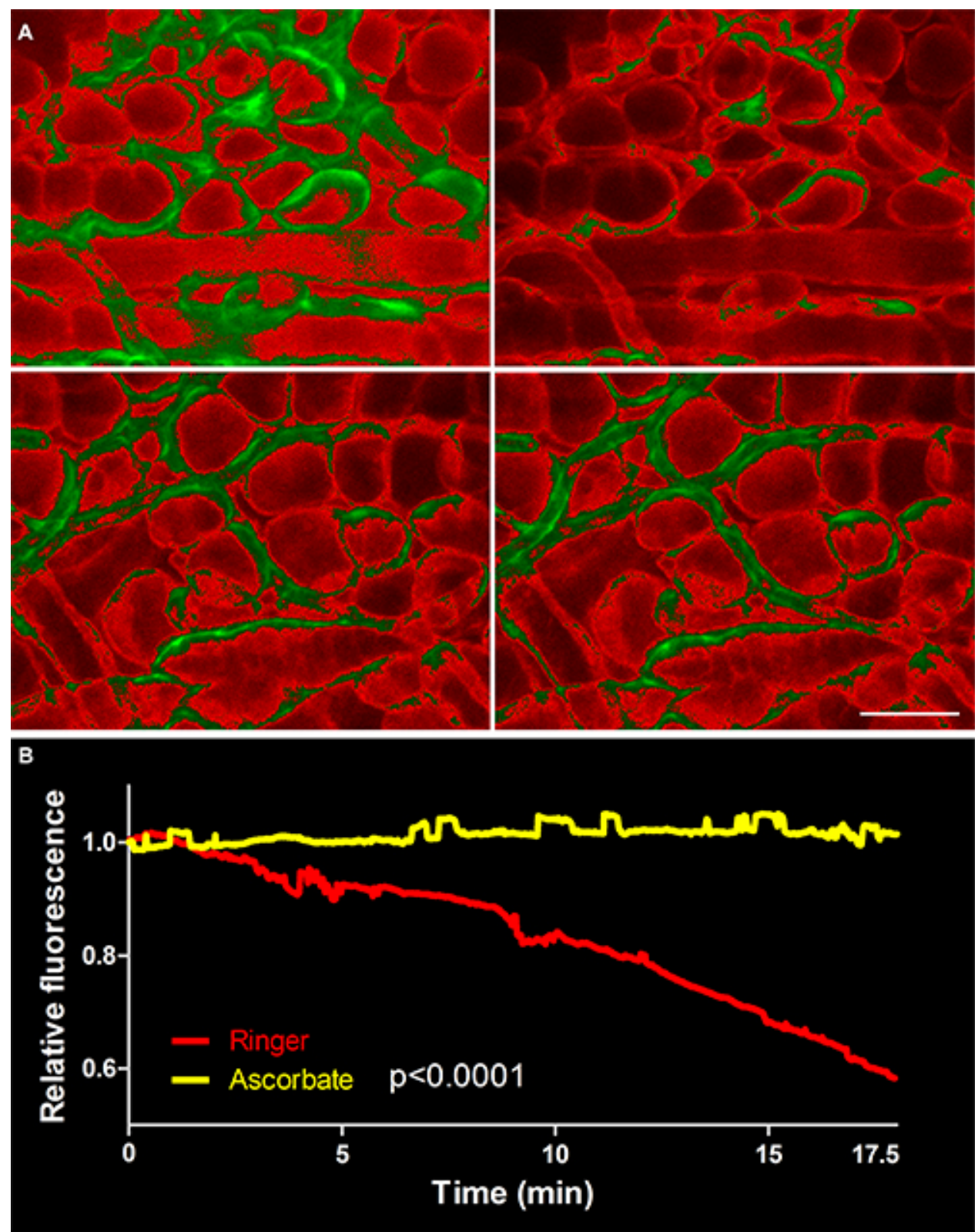

Figure 1. Photobleaching and phototoxicity was stopped by replacing chloride with ascorbate in Ringer's buffer and embedding the exposed ear in $100 \mu \mathrm{l}$ volume of that solution. (A) Tissue was stained first with a biotinylated antibody against collagen IV, the component of the basement membrane. The staining was later detected with streptavidin-647 (red), and then constantly imaged for 300 seconds in either normal Ringer's buffer (upper panel) or ascorbate-Ringer's (lower panel). Note that 300 seconds constant imaging time corresponds to 10 hours of imaging when pictures are collected for $500 \mathrm{msec}$ every one minute (the usual imaging settings). The brightest $25 \%$ of pixel intensity values are green colored. (B) Quantification of immunofluorescence decay (50\% in Ringer's vs. 0\% in ascorbate-Ringer's). Values were normalized to the initial fluorescence. Images collected with immunofluorescence stereomicroscope. Scale bar in A, $100 \mu \mathrm{m}$. Please click here to view a larger version of this figure. 
Investigating the interaction between tumor metastatic cells and tumor stroma is crucial to understand the process of tumor cell migration and tumor immunity. This is because the tumor-associated stroma composes up to $90 \%$ of the tumor mass and actively regulates tumor growth and metastatic spread ${ }^{21}$. However, mechanistic insight into how the matrix drives tumor cell migration towards either lymphatic or blood vessels is lacking ${ }^{22}$. This is partially due to the fact that intravital imaging of matrix proteins is limited to the visualization of matured fibers of fibrillar collagens using second harmonic generation in two-photon microscopy ${ }^{23}$. Therefore, we adapted our method for the visualization of the tissue microenvironment including blood and lymphatic vessels, pericytes, nerves, muscle and adipocytes to include the direct visualization of extracellular matrix components. Many structures can be distinguished based on their morphology after immunostaining for basement membrane components such as collagen IV or perlecan (Fig. 2A), however, direct staining for specific cell surface markers, e.g. Lyve1 (Fig. 2B and C) and podoplanin (Fig. 2C), further allows distinguishing initial, capillary lymphatics (Lyve $1^{+}$) from lymphatic collectors (Lyve $1^{-}$). Staining for matrixbound CCL21 in the skin revealed deposits of this chemokine on the basement membrane of lymphatic collectors identified with the staining for perlecan, the heparan sulphate proteoglycan (Fig. 2D).
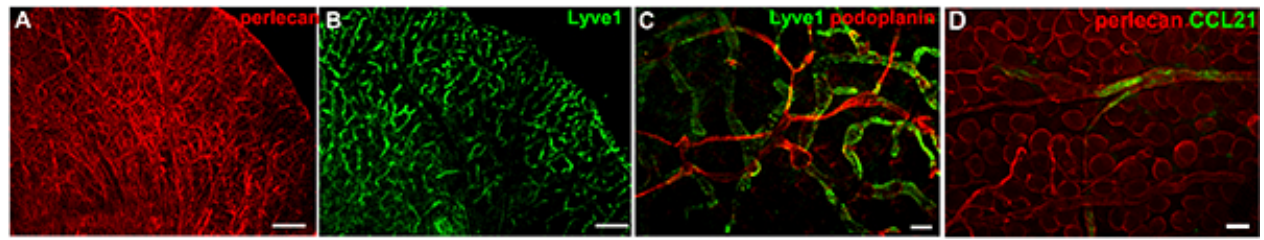

Figure 2. Examples of live staining in a normal mouse ears. (A) Staining for perlecan, a basement membrane component, depicts all blood and lymphatic vessels, nerves, muscle fibers and adipocytes. (B) Lyve1 staining marks the initial lymphatic capillary network. (C) Co-staining for Lyve1 and podoplanin, a pan-lymphatic marker, depicts networks of initial and collecting lymphatics. The dorsal ear dermis can be imaged using classic epifluorescence microscopy (without optical sectioning) as the ear dermis has low number of adipocytes that would otherwise cover the imaging field. (D) CCL21 staining (green) on lymphatic basement membrane stained for perlecan (red). Images collected with immunofluorescence stereomicroscope. Scale bars in A and B, $1 \mathrm{~mm}$; in C, $100 \mu \mathrm{m}$ and $50 \mu \mathrm{m}$ in $\mathbf{D}$. Please click here to view a larger version of this figure.

Most importantly, structural proteins that normally cannot be detected by SHG, the classic matrix detection method ${ }^{23}$, can be visualized. For example, we found that tenascin C (Fig. 3A), a matrix protein that is expressed during tumorigenesis, wound healing and inflammation ${ }^{19}$ is deposited in different locations of tumor stroma than fibrillar collagens (Fig. 3B). This matrix heterogeneity might affect tumor cell distribution and metastasis (Fig. 3C).
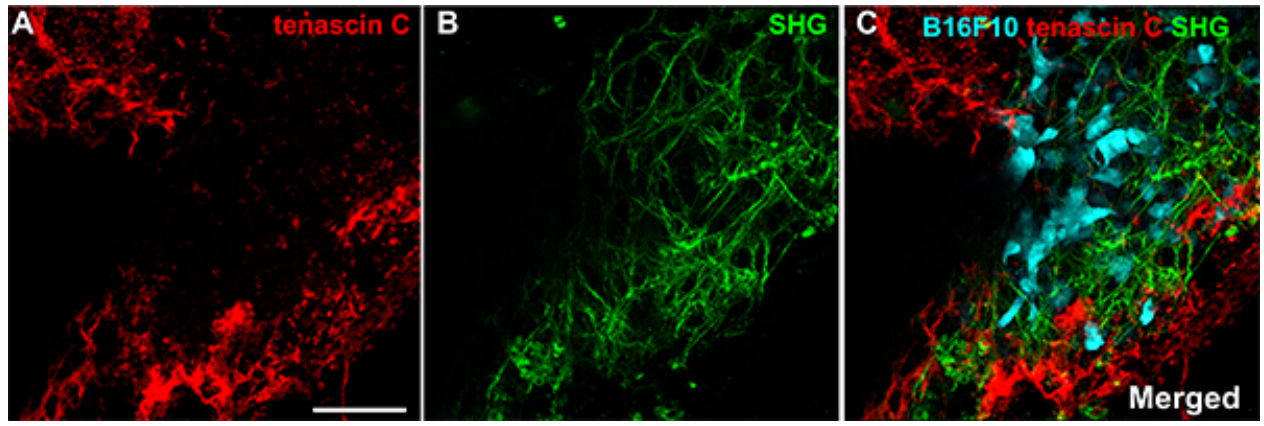

Figure 3. The dorsal ear dermis can be live imaged using multi-photon microscopy. A single field of tumor B16-F10. Tumor stroma was labeled with tenascin $\mathrm{C}$ antibody and the staining was detected with anti-goat-594 donkey antibody. Immunofluorescence of tenascin $\mathrm{C}$ (red) network is shown in $\mathbf{A}$ and fibrillar collagens detected with second harmonic generation (SGH) in $\mathbf{B}$. These two networks are superimposed with tumor cells (cyan) in $\mathbf{C}$ (merged). New tumor matrix marked by tenascin $\mathrm{C}$ is not overlapping with fibrillar collagens detected with SHG (green). The image with 44, four times averaged z-sections with z-step $1.0 \mu \mathrm{m}$ was acquired in 16 bit color depth mode. Images collected with two-photon microscope. Scale bar, $100 \mu \mathrm{m}$. Please click here to view a larger version of this figure.

Following the immunolabeling of different tumor matrix components (e.g. tenascin C and collagen IV) of tumor matrix we could identified restricted and sudden events of tumor matrix directional remodeling (Video 1). Forces that develop within tumor microenvironment may lead to expansion or contraction of the tumor matrix and in consequence remodeling and elongation of the tumor vasculature to a similar extent as we showed previously in the case of healing wounds ${ }^{11,24}$

Video 1. Expansion of tumor matrix labeled with collagen IV (red) and tenascin C (cyan) elongate blood vessels (arrow) and passively translocate three tumor cells (green). Localized contraction of teascin C-rich tumor matrix translocate blood vessel (red horizontally oriented structure) by approximately $100 \mu \mathrm{m}$ during 12 hours of imaging. Images collected with immunofluorescence stereomicroscope. Click here to view video.

Using multi-photon microscopy with fluorophore labeling is problematic as photon flux used in these experiments will quickly bleach fluorescent dyes that are not protected from oxidation ${ }^{25}$. Here we show that we can perform two-photon time-lapse microscopy while simultaneously imaging immunolabeled tenascin C matrix with minimal photobleaching of fluorophores even in high photon density two-photon microscopy (Video 2).

Video 2. Low level photobleaching of tenascin C immunofluorescence (red) during two-photon microscopy. B16-F10-GFP tumor cells (cyan) were imaged in the open dorsal ear 9 days after inoculation. The fluorescent signal was protected by application of ascorbate-Ringer's buffer onto the imaged tissue. Second harmonic generation (green) is not overlapping with new matrix represented by tenascin C. 16 bit color 
depth image with 11 , four times averaged z-sections with z-step $1.9 \mu \mathrm{m}$ was acquired for $15 \mathrm{~min}$. Images were collected every 1 minute 5 seconds in 6 z-planes collected. Click here to view video.

We also imaged immune-cell interactions with metastatic tumor cells. CMTPX-labeled splenocytes from a tumor-bearing mouse extravasated from tumor-associated blood vessels 8 hours after i.v. transfusion, invaded the tumor matrix and actively interacted with tumor cells by forming long-lasting cell contacts (Video 2). Fluorophore-647-labeled collagen IV structures were resistant to photobleaching during the 12 hours of imaging.

Video 3. Interaction of CMTPX-labeled splenocytes from tumor (B16-F10)-bearing mouse with individual tumor cells (GFP- B16-F10). Splenocytes were i.v. transfused after tissue staining for collagen IV. Collagen IV-green (647), splenocytes-red (CMTPX), B16-F10 cyan (GFP). Images collected with immunofluorescence stereomicroscope. Video duration 12 hours. Click here to view video.

Freshly isolated tumor cells were overlaid on the exposed ear dermis pre-stained for collagen IV. We could observed that after adhering to the tissue some groups of cells started collective migration along basement membrane of blood vessels and adipocytes.

Video 4. Tumor cells migrate along basement membranes. Normal ear skin stained for collagen IV (647, red) was overlaid with freshly passaged B16-F10-GFP cells and imaged for 5 hours. Some tumor cells that adhered to tissue started collective migration along basement membrane of blood vessels and adipocytes. Images collected with immunofluorescence stereomicroscope. Video duration: 5 hours. Click here to view video.

Also, because the ear dermis is virtually two-dimensional, we could readily collect large volumes of data using fast fluorescence stereomicroscopy, instead of slower and more expensive confocal or multiphoton microscopy. However, it is also possible to use any of the mentioned scanning microscopy methods with our intravital IF preparations (Fig. 3, Video 1).

\section{Discussion}

\section{Significance}

Here we present a novel intravital microscopy approach that allows high resolution and dynamic visualization of different tissue microenvironment components, including fibrillar as well as mesh-like matrix proteins. This method has several advantages over current intravital imaging techniques: (i) Intravital fluorescence imaging has been used in microcirculation studies, e.g., to track solute leakage from blood or into lymphatics, but has not been combined with immunostaining. (ii) The use of genetically modified reporter mice allows for specific cell types to be imaged, but requires their availability (or substantial effort to create new ones) and limits the number of interacting cell types that can be studied. (iii) Extracellular matrix can be imaged in vivo using second harmonic generation, but this technique can only detect fibrous collagens, leaving a large number of important extracellular components such as basement membranes, fibronectins, tenascins, growth factors, chemokines and tissue glycosaminoglycans out of reach for current research. Our method overcomes these limitations, and allows standard imaging techniques to be incorporated and further combined with immunostainings for other cell types, tissue structures, deposits of heparin sulfate binding growth factors (e.g. VEGF ${ }^{26}$ ) and chemokines (CCL2 $1^{5,18}$ and Fig. 2D), or extracellular matrix proteins while simultaneously tracking blood and/or lymphatic flows.

\section{Limitations}

The epifluorescence imaging of intravital IF is limited to the thin skin flaps, deprived of thick hypodermis (adipose tissue). While we found that the dorsal ear dermis is optimal for relatively harmless surgical exposition of the ear skin, epifluorescence with intravital IF technique is not limited to ear dermis and could potentially be applied to e.g. the exposed skin of the toes or the back skin of the newborn mouse. Rapid antibody staining (15 minutes) in immunofluorescence IF is not dependent on passive diffusion but requires functional lymphatic drainage and interstitial fluid flow, hence no staining can be observed if lymphatic vessels are occluded ${ }^{27}$. In line with that, lymphatic vessels stain stronger then leaky blood vasculature (injured vessels, arterioles) ${ }^{5}$. The separation of the dorsal and ventral ear skin flaps is a mild surgical procedure but it causes injury to some capillaries and cell death to various tissue cells. This might be a problem when one needs to investigate completely intact tissue, e.g. looking at the mild effect of drugs on cell survival. Also the application of antioxidant that serves to protect the skin from phototoxicity and photobleaching excludes the use of intravital immunofluorescence technique to study e.g. the tissue oxidative stress or nitric oxide biology.

Finally, blinding of tissue resident macrophages with immune complexes can activate these cells and influence e.g. the study of tissue immune response and dendritic cell activation.

\section{Modifications and troubleshooting}

In order to study tissue oxidative stress or nitric oxide biology, ascorbate has to be replaced with other tissue compatible media that does not interfere with the experimental output. Similarly, blocking Fc receptors on dermal immune cells should be avoided if the goal of the study is the function and activation of the tissue immune response and dendritic cell activation and migration. This can be done with the use of secondary antibody with $\mathrm{Fc}$ fragment cleaved $(\mathrm{F}(\mathrm{ab}) 2$ antibody fragments) or the use of biotinylated antibody and fluorescent streptavidin as detection reagents.

\section{Future applications}

We present a novel and unique intravital IF technique to image non-fibrillar components of extracellular matrix in transplantable skin tumors in combination with SHG-detected fibrillar and matured collagens using two-photon microscopy. One of the advantages of using multi-photon (or confocal) microscopy over epifluorescence imaging is z-stacking possibility and in consequence spatial co-localization of events occurring within extracellular matrix, e.g. tumor intravasation into lymphatic or blood vessel, which in the case of standard epifluorescence microscopy can be only inferred from morphological changes of the invading cell. This technique has far broader potential. For example, we have used the intravital IF to investigate the mechanism of lymphatic occlusion by lymphatic-specific photodynamic therapy ${ }^{27}$. Additional potential applications of this 
method include but are not limited to investigation of skin immunity during inflammation, mechanism of transplant rejection or methods of blood and lymphatic vessel growth during tumorigenesis.

\section{Critical steps in the procedure}

The most important step that has critical implications for sustaining a physiological tissue environment is a surgical separation of the ventral skin and cartilage from dorsal dermis. Cutting or occluding the major artery or vein will lead to excessive bleeding and local tissue hypoxia, which will affect cellular response to treatments and cell movement ${ }^{8}$. Immobilizing the ear with surgical glue should be done with care as spilling the glue on expose tissue will cause a permanent injury to the tissue by occlusion of blood vessels. The temperature of the mouse must be controlled and kept at $37^{\circ} \mathrm{C}$. Additionally, humidified oxygen needs to be used for the isoflurane anesthesia, so that the eyes and lungs stay properly humidified during the experiment. Also, sodium ascorbate must be prepared freshly and its $\mathrm{pH}$ checked before use.

In summary, this intravital immunofluorescence bridges real-time, local measurements of physiological function with molecular imaging of complex cellular events in the mouse skin. Furthermore, this method has great potential as it can be easily applied to e.g. study postdevelopmental mechanisms of blood and lymph-angiogenesis or to visualize the early phases of skin infection by various pathogenic agents. With its flexibility and high-throughput potential, this intravital technique can significantly contribute to multiple fields of biology.

\section{Disclosures}

The authors have nothing to disclose.

\section{Acknowledgements}

The authors are grateful to Jeremy C. M. Teo and S. Ryan Oliver for their contribution and Jolanta Kilarska for help in image processing. We thank the BIOP core facility at EPFL for the support with two-photon microscopy

This work was supported in part by grants from the European Research Commission (DC-Lymph, 206653-2), the European Framework Project 7 (AngioScaff), the Swiss National Science Foundation (31-135756), and the U.S. National Institutes of Health (NIH)/NIH Heart, Lung, and Blood Institute (NHLBI) (RO1 HL096539). Additionally, funds from the Robert Wenner Award (Swiss Cancer League) allowed the purchase the Leica stereomicroscope used in this study. The funders had no role in study design, data collection and analysis, decision to publish, or preparation of the manuscript.

\section{References}

1. Martinez-Corral, I., et al. In vivo imaging of lymphatic vessels in development, wound healing, inflammation, and tumor metastasis. Proc Natl Acad Sci USA, doi:10.1073/pnas.1115542109 (2012).

2. Snippert, H. J., et al. Intestinal crypt homeostasis results from neutral competition between symmetrically dividing Lgr5 stem cells. Cell. 143 , 134-144, doi:10.1016/j.cell.2010.09.016 (2010).

3. Halin, C., Mora, J. R., Sumen, C., \& von Andrian, U. H. In vivo imaging of lymphocyte trafficking. Annu. Rev. Cell Dev. Biol. 21, 581-603, doi:10.1146/annurev.cellbio.21.122303.133159 (2005).

4. Kilarski, W., Petersson, L., Fuchs, P., Zielinski, M., \& Gerwins, P. An in vivo neovascularization assay for screening regulators of angiogenesis and assessing their effects on pre-existing vessels. Angiogenesis. 15, 643-655, doi:10.1007/s10456-012-9287-8 (2012).

5. Kilarski, W. W., et al. Intravital Immunofluorescence for Visualizing the Microcirculatory and Immune Microenvironments in the Mouse Ear Dermis. PLoS ONE. 8, e57135, doi: doi:10.1371/journal.pone.0057135 (2013)

6. Steinbauer, M., et al. GFP-transfected tumor cells are useful in examining early metastasis in vivo, but immune reaction precludes long-term tumor development studies in immunocompetent mice. Clin. Exp. Metastasis. 20, 135-141 (2003).

7. Ohashi, T., Kiehart, D. P., \& Erickson, H. P. Dynamics and elasticity of the fibronectin matrix in living cell culture visualized by fibronectingreen fluorescent protein. Proc Natl Acad Sci U S A. 96, 2153-2158 (1999).

8. Egeblad, M., et al. Visualizing stromal cell dynamics in different tumor microenvironments by spinning disk confocal microscopy. Dis. Models Mech. 1, 155-167, doi:10.1242/dmm.000596 (2008).

9. Tal, O., et al. DC mobilization from the skin requires docking to immobilized CCL21 on lymphatic endothelium and intralymphatic crawling. $J$. Exp. Med. 208, 2141-2153, doi:10.1084/jem.20102392 (2011).

10. Roos, A., Trouw, L. A., loan-Facsinay, A., Daha, M. R., \& Verbeek, S. J. in Encyclopedia of Life Sciences 1-14, John Wiley \& Sons (2005).

11. Kilarski, W. W., Samolov, B., Petersson, L., Kvanta, A., \& Gerwins, P. Biomechanical regulation of blood vessel growth during tissue vascularization. Nat Med. 15, 657-664 (2009).

12. Sahai, E., et al. Simultaneous imaging of GFP, CFP and collagen in tumors in vivo using multiphoton microscopy. BMC Biotechnol. 5(1), 14, doi:10.1186/1472-6750-5-14 (2005).

13. Wyckoff, J. B., Jones, J. G., Condeelis, J. S., \& Segall, J. E. A critical step in metastasis: In vivo analysis of intravasation at the primary tumor. Cancer Res. 60, 2504-2511 (2000).

14. Pinner, S., \& Sahai, E. Imaging amoeboid cancer cell motility in vivo. J. Microsc. 231, 441-445, doi:10.1111/j.1365-2818.2008.02056.x (2008).

15. Lämmermann, T., et al. Rapid leukocyte migration by integrin-independent flowing and squeezing. Nature. 453, 51-55, doi:10.1038/ nature06887 (2008).

16. Padera, T. P., Stoll, B. R., So, P. T., \& Jain, R. K. Conventional and high-speed intravital multiphoton laser scanning microscopy of microvasculature, lymphatics, and leukocyte-endothelial interactions. Mol. Imaging. 1, 9-15 (2002).

17. Giampieri, S., et al. Localized and reversible TGFbeta signalling switches breast cancer cells from cohesive to single cell motility. Nat. Cell Biol. 11, 1287-1296, doi:10.1038/ncb1973 (2009).

18. Weber, M., et al. Interstitial dendritic cell guidance by haptotactic chemokine gradients. Science. 339, 328-332, doi:10.1126/science.1228456 (2013). 
19. Midwood, K. S., \& Orend, G. The role of tenascin-C in tissue injury and tumorigenesis. Journal of cell communication and signaling. $\mathbf{3}$, 287-310, doi:10.1007/s12079-009-0075-1 (2009).

20. Pawley, J. B. in Handbook of Biological Confocal Microscopy. Ch. 340-341, Springer (2006).

21. Sund, M., \& Kalluri, R. Tumor stroma derived biomarkers in cancer. Cancer Metastasis Rev. 28, 177-183, doi:10.1007/s10555-008-9175-2 (2009).

22. Mueller, M. M., \& Fusenig, N. E. Friends or foes - bipolar effects of the tumour stroma in cancer. Nat Rev Cancer. 4, 839-849 (2004).

23. Brown, E., et al. Dynamic imaging of collagen and its modulation in tumors in vivo using second-harmonic generation. Nat. Med. 9, 796-800, doi:10.1038/nm879 (2003).

24. Rice, J. J., Gerwins, P., \& Kilarski, W. W. Mechanisms of Angiogenesis: Perspectives from Antiangiogenic Tumor Therapies. Current Angiogenesis. 1, 139-147 (2012).

25. Patterson, G. H., \& Piston, D. W. Photobleaching in two-photon excitation microscopy. Biophysical journal. 78, 2159-2162, doi:10.1016/ S0006-3495(00)76762-2 (2000).

26. Jakobsson, L., et al. Heparan sulfate in trans potentiates VEGFR-mediated angiogenesis. Dev Cell. 10, 625-634, doi:10.1016/ j.devcel.2006.03.009 (2006).

27. Kilarski, W. W., et al. Optimization and regeneration kinetics of lymphatic-specific photodynamic therapy in the mouse dermis. Angiogenesis. Ahead of print, doi:10.1007/s10456-013-9365-6 (2013). 